\title{
Mitochondrial ultrastructure-associated chemotherapy response in ovarian cancer
}

\author{
MOTOAKI SAITOU $^{1}$, SEIJI ISONISHI ${ }^{1}$, TOMOMI HAMADA ${ }^{2}$, TAKAKO KIYOKAWA ${ }^{2}$, \\ TOSHIAKI TACHIBANA ${ }^{3}$, HIROSHI ISHIKAWA ${ }^{3}$ and MAKOTO YASUDA ${ }^{1}$ \\ Departments of ${ }^{1}$ Obstetrics/Gynecology, ${ }^{2}$ Pathology and ${ }^{3}$ Anatomy, Jikei University \\ School of Medicine, 3-25-8 Nishi-shinbashi, Minato-ku, Tokyo 105, Japan
}

Received July 26, 2008; Accepted September 26, 2008

DOI: $10.3892 /$ or_00000208

\begin{abstract}
We developed mitochondrial (MT) scoring system based on MT ultrastructural findings in association with response to chemotherapy in ovarian cancer (OC). Ultrathin sections of MT prepared from 28 OC patients before chemotherapy were examined by electron microscopy. Platinum-sensitive ovarian carcinoma cell line 2008 and its resistant variant $\mathrm{C} 13$ were used as control cells. Seven independent MT features including, diameter, pattern of cresta structure, electron density, distribution-density and pattern, ratio of minimal/maximal diameter and MT architecture were examined and were assigned a score between 0 and 2 . Twenty-eight cases were primary advanced OC, including 4 recurrent cases. Nine cases were chemosensitive while 19 were resistant. Univariate and multivariate analysis in each factor showed good correlation to chemosensitivity for 2 factors of electron density, distribution pattern. Total score of these 2 factors in 9 sensitive cells was $1.44 \pm 0.41(\mathrm{M} \pm \mathrm{SE})$ and was $3.58 \pm 0.18$ in 19 resistant cells $(\mathrm{P}<0.001)$. Receptor operative characteristics (ROC) analysis revealed that total 'cut-off' score was 3 point $(\mathrm{P}<0.05$; AUC $=0.84)$. In conclusion, this MT scoring system was excellently correlated to response regardless of histopathologic findings and this strongly suggests that the system is deemed to be of great value as biomarker for the chemosensitivity in OC.
\end{abstract}

\section{Introduction}

Platinum drugs remain one of the most important chemotherapeutic agents for ovarian cancer treatment. Resistance to cisplatin is a major obstacle for successful cancer therapy. Although significant biochemical changes in

Correspondence to: Dr Seiji Isonishi, Department of Obstetrics/ Gynecology, Jikei University Aoto Hospital, 6-41-2 Aoto, Katsushika-ku, Tokyo 125-8506, Japan

E-mail: isonishi@jikei.ac.jp

Key words: mitochondrial ultrastructure, cisplatinum sensitivity, ovarian carcinoma the cisplatin-resistant cells have been described, the description of the mechanisms resulting in cellular cisplatin resistance remains unclear. Although formation and repair of cisplatin nuclear-DNA adducts (1) and inhibition of DNA synthesis have been widely studied, several lines of evidence showed that platinum nuclear-DNA interaction was not the core effect of cisplatin (2). Furthermore, it was shown that tumor cell exposure to cisplatin ultimately results in apoptosis (3). Only $1 \%$ of intracellular platinum is bound to nuclearDNA, with the great majority of the intracellular drug available to interact with other molecules, including mitochondrial (MT) DNA (4). Our Bcl-2 data (5) suggested the importance of mitochondrial pathways in cisplatin action; we have subsequently centered our attention on cisplatin interactions with MT and its DNA (6).

A considerable number of reports have also shown the ability of various compounds to act directly on mitochondria, inducing loss of membrane potential and release of apoptogenic proteins from isolated mitochondria $(7,8)$. We also reported the MT cytochrome c release-associated enhancement of platinum sensitivity in ovarian carcinoma cells (9). In the current study, we also report the morphological changes in association with platinum sensitivity.

The histopathologic findings of ovarian epithelial carcinoma have generally been found to be of prognostic significance concerning response to chemotherapy. Serous adenocarcinoma is considered to be most sensitive while other types are less sensitive to standard chemotherapeutic regimens. Previously, Silverberg has reported the prognostic significance of a histopathologic grading system of ovarian epithelial carcinoma (10). In this stydy he emphasized that histopathologic typing is less valuable than grading in predicting survival but better at predicting tumor responsiveness to chemotherapy, and can also suggest the chemotherapeutic agents to be used. On the other hand the pathological features of ovarian cancer after chemotherapy have been detailed previously (11). As to the ultrastructural features of MT in ovarian cancer, there have been no particular studies reported. A pathologic textbook showed typical MT findings (12), but the significance of MT morphology remained unclear.

The purpose of this study was to develop a scoring system of MT morphological findings to determine whether there is a relation between ultrastructural findings and 
Table I. Scoring system of mitochondrial ultrastructure.

\begin{tabular}{|c|c|c|c|}
\hline Point & 0 & 1 & 2 \\
\hline $\begin{array}{l}\text { MT size } \\
\quad \text { (Longest diameter) }\end{array}$ & $\begin{array}{c}\text { Small } \\
(\leq 0.7 \mu \mathrm{m})\end{array}$ & Intermediate & $\begin{array}{c}\text { Large } \\
(\geq 0.8 \mu \mathrm{m})\end{array}$ \\
\hline Cresta structure & Clear & Intermediate & Destroyed \\
\hline Electron density & Low & Intermediate & High \\
\hline $\begin{array}{l}\text { MT distribution } \\
\qquad\left(100 \mu \mathrm{m}^{2}\right)\end{array}$ & $\begin{array}{c}\text { High } \\
\left(\geq 80 / 100 \mu \mathrm{m}^{2}\right)\end{array}$ & Intermediate & $\begin{array}{c}\text { Low } \\
\left(\leq 40 / 100 \mu \mathrm{m}^{2}\right)\end{array}$ \\
\hline Distribution pattern & Perinuclear & Intermediate & Dispersed \\
\hline $\begin{array}{l}\text { Oval ratio } \\
\text { (Short/long) }\end{array}$ & $\begin{array}{l}\text { Short ovoid } \\
\qquad(\geq 0.7)\end{array}$ & Intermediate & $\begin{array}{l}\text { Long ovoid } \\
\qquad(\leq 0.3)\end{array}$ \\
\hline MT type & Tubular & & Adrenal/hepatocyte \\
\hline
\end{tabular}

MT Type

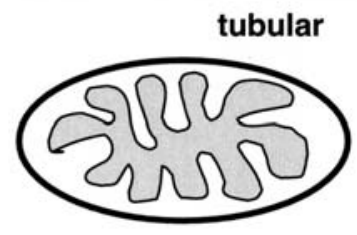

responsiveness to chemotherapy with analysis of application of this system to a series of cases treated in a uniform manner in our institution.

\section{Materials and methods}

Patients. The study group comprised 28 women with advanced stage or recurrent ovarian cancer who had been treated with primary surgery followed by taxane- plus platinum-based chemotherapy at the Department of Obstetrics and Gynecology of Jikei University School of Medicine, Japan between January 2000 and January 2006. Patients were enrolled onto the study after providing informed consent. The study protocol and all accompanying forms and surveys were reviewed and approved by the institutional review board.

Histologic diagnosis. The tumor stage and histologic diagnosis of each patient were determined according to FIGO criteria and the histologic typing system of the WHO, respectively. All available histological sections were reviewed by two expert histopathologists involved in our study. For the grading of tumor, we used the grading system of the Gynecologic Oncology Group (GOG) (13).

Clinical evaluation. The evaluation of the clinical course of disease was based on clinical examination, serum CA125 assay, chest X-ray, abdominal-pelvic ultrasound and computed tomography scan. Additional investigations were performed when appropriate. Response was characterized according to the Response Evaluation Criteria in Solid Tumors (RECIST) criteria (14). When the patient did not have any assessable tumor, CA125 response criteria (Gynecologic Cancer
Intergroup-modified Rustin definition) (15) was used. Serum CA125 values were measured immediately before and after chemotherapy. The best assessed overall response was observed in all patients.

Electron microscopy. For electron microscopic analysis, cells or samples were rinsed and fixed in situ with $2.0 \%$ glutaraldehyde in $0.1 \mathrm{M}$ phosphate buffer with calcium for $1 \mathrm{~h}$ at $4^{\circ} \mathrm{C}$. After washes in $0.1 \mathrm{M}$ phosphate buffer $\mathrm{pH} 7.4$, cells were fixed for $1 \mathrm{~h}$ at $4^{\circ} \mathrm{C}$ in $1 \% \mathrm{OsO}_{4}$. Samples were dehydrated in graded concentrations of ethanol and embedded in Epon 812 epoxy resin. After polymerization, ultrathin sections were cut parallel to the block surface using a Reichert OUM4 ultramicrotome, stained with uranyl acetate and lead citrate, and then examined in a JEOL-1200EX electron microscope at $60 \mathrm{kv}$ acceleration voltage at magnification of x1000, x1200 and x2500.

By careful examination and comparison of MT in typical platinum sensitive and resistant ovarian carcinoma cells, we focused on 7 independent parameters depicting the most prominent differences between the two representative cells. As shown in Table I, these features are evaluated in 10 MTs as follows. i) MT size (longest diameter; $\leq 0.7 \mu \mathrm{m}, 0.7-0.8$, $\geq 0.8 \mu \mathrm{m}$ ); ii) cresta structure (clear, intermediate and destroyed); iii) electron density (low, intermediate and high); iv) MT distribution (number/100 $\mu \mathrm{m}^{2}: \geq 80,40-80, \leq 40$ ); v) distribution of MT pattern (perinuclear, intermediate and dispersed); vi) ovoid ratio (shortest diameter/longest diameter: $\geq 0.7$, intermediate, $\leq 0.3$ ) and vii) MT architecture (3 types of MT shown in Table I: tubular, adrenal or hepatocyte). Each of these evaluated parameters is given a point score 0-2 except for MT architecture which is given 0 
Table II. Patient demographics.

\begin{tabular}{|c|c|c|c|c|c|}
\hline \multirow[b]{2}{*}{ Characteristic } & \multirow[b]{2}{*}{ Total } & \multicolumn{2}{|c|}{ Responder } & \multicolumn{2}{|c|}{ Non-responder } \\
\hline & & No. & $\%$ & No. & $\%$ \\
\hline No. & 28 & 9 & & 19 & \\
\hline Age (median) & 51 & 51 & & 48 & \\
\hline \multicolumn{6}{|l|}{ Stage } \\
\hline Ic recurrence & 4 & 0 & 0.0 & 4 & 21.1 \\
\hline III & 19 & 7 & 77.8 & 12 & 63.2 \\
\hline IV & 5 & 2 & 22.2 & 3 & 15.8 \\
\hline \multicolumn{6}{|l|}{ Histology } \\
\hline Serous & 14 & 8 & 88.9 & 6 & 31.6 \\
\hline Endometrioid & 4 & 0 & 0.0 & 4 & 21.1 \\
\hline Clear cell & 4 & 1 & 11.1 & 3 & 15.8 \\
\hline Undifferentiated & 3 & 0 & 0.0 & 3 & 15.8 \\
\hline Unclassified & 3 & 0 & 0.0 & 3 & 15.8 \\
\hline \multicolumn{6}{|l|}{ Tumor grade } \\
\hline 1 & 5 & 0 & 0.0 & 5 & 50.0 \\
\hline 2 & 5 & 2 & 25.0 & 3 & 30.0 \\
\hline 3 & 8 & 6 & 75.0 & 2 & 20.0 \\
\hline \multicolumn{6}{|l|}{ Max. tumor size } \\
\hline$\leq 1 \mathrm{~cm}$ & 19 & 7 & 77.8 & 12 & 63.2 \\
\hline$>1 \mathrm{~cm}$ & 9 & 2 & 22.2 & 7 & 36.8 \\
\hline \multicolumn{6}{|c|}{ No. of residual lesions } \\
\hline$\leq 5$ & 19 & 7 & 77.8 & 12 & 63.2 \\
\hline$>5$ & 9 & 2 & 22.2 & 7 & 36.8 \\
\hline
\end{tabular}

${ }^{\mathrm{a}}$ Grading for serous, endometrioid and clear cell types and ${ }^{\mathrm{b}}$ maximum size of the residual tumor.

or 2. Scoring was done by discussion of two reviewers; one gynecologist with an assistance, or one anatomist. Fig. 1A and B illustrate typical examples of the patterns used assigning the point score for MT characteristics of OC in this system. Fig. 1A demonstrates typical MT in drug sensitive OC cells, showing lower electron density, perinuclearly distributed, clear cresta structure. Fig. 1B depicts MT in drug resistant OC cells, showing higher electron density, dispersed distribution pattern, unclear cresta structure.

Statistical analysis. Differences between samples or groups of samples were determined by Student's t-test using twosided P-values. Multiple logistic regression was carried out to investigate the relationship among the response and the factors. Receiver operator characteristics (ROC) were performed to determine if an optimal 'cut-off' point could be determined for MT scoring system and subsequent drug sensitivity.

\section{Results}

Patient characteristics. Patient demographics are summarized in Table II. The median age of patients was 51 years (range
27-68). According to the FIGO classification, tumor stage was III in 19 patients, IV in 5 patients, and recurrence in 4 patients. In all recurrent cases, time intervals from the last day of primary chemotherapy to the start of secondary chemotherapy were $>6$ months. Histologically, 14 carcinomas were serous, 4 was endometrioid, 4 were clear cell, 3 were undifferentiated and the rest of 3 were unclassified adenocarcinoma. Out of 6 chemotherapy non-responders of serous adenocarcinoma, 4 were recurrent cases. Tumor was graded for serous and endometrioid carcinoma and the grade was G1 in 5 patients, G2 in 5, and G3 in 8 . All 4 endometrioid carcinoma were G1, while 6 out of $8 \mathrm{G} 3$ cases were primary serous carcinoma. After the surgery, 19 patients had residual disease $\leq 1 \mathrm{~cm}$ and 9 patients had a larger residual tumor. Chemotherapy response did not relate to stage, histology, grade, tumor size or residual lesion.

Scoring system for mitochondrial ultrastructure. Seven factors were analyzed and scored independently in our grading system (Table III). With the exception of MT size, six selected factors were found to work significantly as independent indicators for chemosensitivity $(\mathrm{P}<0.05)$. Multivariate analysis of these selected 6 factors revealed that electron 


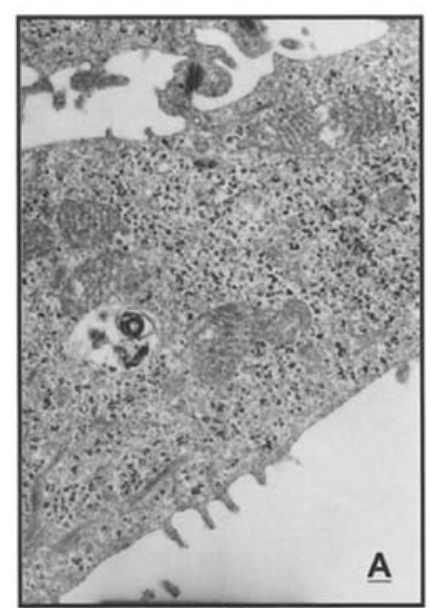

Platinum Sensitive

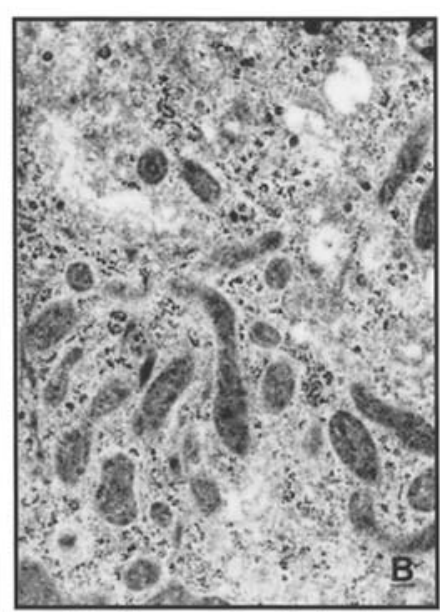

Platinum Resistant $(\times 15 \mathrm{~K}-; 500 \mathrm{~nm})$

Figure 1. Representative electron microscopy of mitochondria of typical drug sensitive (A) and resistant (B) ovarian carcinoma cells, x1500. Scoring are defined in text and Table I. (A) Longest MT size is $0.59 \pm 0.12 \mu \mathrm{m}(0$ point), cresta structure is clearly visible (0 point), electron-density is very low (0 point), MT-distribution is $100.9 \mathrm{count} / \mu \mathrm{m}^{2}$ ( 0 point), perinuclear MT distribution pattern ( 0 point), short ovoid ratio (shortest diameter/longest diameter; $0.81 \pm 0.17)(0$ point $)$, tubular MT type $(0$ point $)$. Total score $=0$ point. (B) Longest MT size is $0.89 \pm 0.09 \mu \mathrm{m}$ (2 point), cresta structure is destructive ( 2 point), electron-density is very high (2 point), MT-distribution is 39.7 count $/ \mu \mathrm{m}^{2}$ (2 point), dispersed MT distribution pattern (2 point), long ovoid ratio (shortest diameter/longest diameter; $0.16 \pm 0.04$ ) (2 point), hepatocyte MT type ( 2 point). Total score $=14$ point.

density and MT distribution pattern are the 2 major independent factors (Table IV).

Since each of these 2 parameters proved to be independent of one another, and because each of them correlated in univariate analysis with chemosensitivity, it was deemed appropriate to combine them to create our final scoring system (Table IV). As mentioned above, each parameter is scored from 0 to 2 , and the scores are then added, with a final score from 0 to 4 yielding a final sensitivity classification of sensitive (0-2), 3-4 being classified as resistant. This cut-off point of $\geq 3$ as resistant was determined by ROC analysis

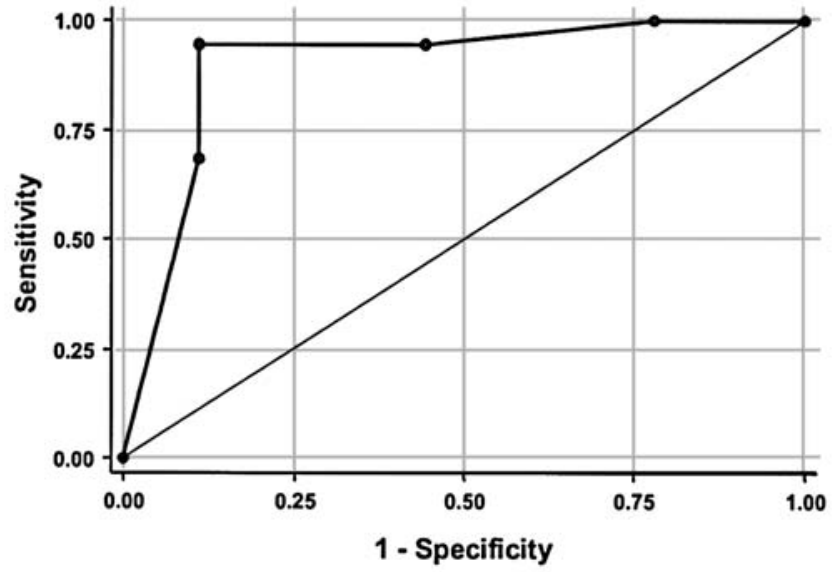

Figure 2. Receiver operator characteristics (ROC) was performed to determine the optimal 'cut-off' score of $\geq 3$ as resistant with sensitivity of $94.7 \%$ and specificity of $88.9 \%$.

Table III. Results of scoring system.

\begin{tabular}{lccc}
\hline Characteristic & $\begin{array}{c}\text { Sensitive }(\mathrm{N}=9) \\
\text { Mean } \pm \mathrm{SE}\end{array}$ & $\begin{array}{c}\text { Resistant }(\mathrm{N}=19) \\
\text { Mean } \pm \mathrm{SE}\end{array}$ & $\mathrm{P}$ \\
\hline MT size & $1.000 \pm 0.289$ & $1.421 \pm 0.159$ & 0.0886 \\
Cresta structure & $0.778 \pm 0.324$ & $1.685 \pm 0.134$ & 0.0024 \\
Electron density & $0.556 \pm 0.242$ & $1.833 \pm 0.090$ & $<0.0001$ \\
MT distribution & $0.667 \pm 0.289$ & $1.556 \pm 0.121$ & 0.0012 \\
Distribution pattern & $0.889 \pm 0.261$ & $1.842 \pm 0.086$ & 0.0001 \\
Ovoid ratio & $0.222 \pm 0.222$ & $0.944 \pm 0.205$ & 0.0195 \\
MT type & $1.111 \pm 0.351$ & $1.889 \pm 0.111$ & 0.0065 \\
Total & $5.222 \pm 1.451$ & $10.842 \pm 0.632$ & 0.0001 \\
\hline
\end{tabular}

measuring success as drug sensitivity (AUC=0.90) (Fig. 2). Group mean comparison test for this new scoring system showed the statistically significant difference with the total score of $1.44 \pm 0.41$ for sensitive group (mean $\pm \mathrm{SE}$ ) vs.

Table IV. Multivariate analysis of 6 selected factors.

\begin{tabular}{lccccc}
\hline & & & & \multicolumn{2}{c}{$95 \% \mathrm{CI}^{\mathrm{a}}$} \\
\cline { 5 - 6 } Characteristic & Coefficient & $\mathrm{t}$ & $\mathrm{P}$ & Lower & Upper \\
\hline Cresta structure & 0.083 & 1.66 & 0.555 & -0.374 & 0.207 \\
Electron density & 0.367 & 2.77 & 0.012 & 0.091 & 0.644 \\
MT distribution & 0.121 & 0.95 & 0.352 & -0.385 & 0.144 \\
Distribution pattern & 0.370 & 2.53 & 0.020 & 0.065 & 0.675 \\
Ovoid ratio & 0.086 & 1.00 & 0.331 & -0.268 & 0.095 \\
MT type & 0.137 & 0.98 & 0.337 & -0.154 & 0.427 \\
\hline Electron density + & & & & 1.354 & 2.914 \\
Distribution pattern & & 5.62 & $<0.001$ & & \\
\hline
\end{tabular}

a95\% Confidence interval. 
$3.58 \pm 0.18$ for resistant $(\mathrm{P}<0.0001)$. The system works in primary and recurrent cases with ovarian carcinoma of the major histologic types (serous, clear cell, endometrioid), as well as in primary cases of undifferentiated adenocarcinoma and others.

By univariate analysis for a group of features including histologic tumor type, GOG grade, and this new scoring system, histologic type (primary serous carcinoma) and our system were significantly correlated with chemosensitivity.

\section{Discussion}

Cytoreductive surgery followed by paclitaxel-plus platinum based chemotherapy represents the standard treatment for patients with advanced epithelial OC. A recent randomized study revealed no difference in response rates, progression-free survivals, and early survival data between docetaxel vs. paclitaxel, or carboplatin versus cisplatin $(16,17)$. We highlighted two major questions for this gold standard chemotherapy. First, the majority of the cases in those randomized studies were typical serous adenocarcinoma, the most platinum-sensitive common histologic type and they do not include good sample size for other histologic types of tumor. Second, the use of this combination chemotherapy as secondline for relapse is restricted to cases with long treatment-free interval (TFI) ( $>6$ months) because of the probable acquired platinum resistance. Our unique MT scoring system has addressed these questions and sorted them out.

For the first problem, no one knows whether taxane plus platinum combination chemotherapy is as effective in other histologic types of tumor as in serous adenocarcinoma. Half of the ovarian cancer patients at least in Japan have mucinous, clear cell, and low grade serous ovarian cancers, distinct from the more common high grade serous papillary cancers. There is no previous literature indicating standard chemotherapeutic regimens or biological markers peculiar to those relatively rare tumors. Fourteen of our 28 cases were other than serous OC and $93 \%$ of them were diagnosed as platinum resistant. Our MT scoring system predicted platinumresistance correctly in $92 \%$ cases (positive predictive value = 0.92 ), suggesting the system is a valuable marker for the diagnosis of platinum-resistance in non-serous tumors as well as in typical serous adenocarcinoma. Based on our scoring system, we can recommend to consider chemotherapeutic regimens without platinum for those patients scored as platinum-resistant.

Regarding the second point above, the major problem for the recurrent cases is the complex pathological diagnosis of the recurrent tumor. When the patient relapses after chemotherapy, the surviving tumors show morphological effects of chemotherapy (11). Tumor type is predictive of drug-sensitivity and grading may be a predictor of prognosis for primary tumors, but neither tumor typing nor grading is reliable after chemotherapy. The carcinoma occasionally could not be classified as either serous, mucinous, endometrioid, or clear cell. The treatment-free interval (TFI) has been established as predictive for response to re-treatment with a platinumcontaining regimen, although more recent data indicate it may not be an independent factor (18). In this regard, our MT scoring system could be a powerful tool to predict platinum sensitivity of surviving tumors. Our MT system correctly diagnosed 4 recurrent cases as platinum resistant despite the $>6$ month treatment-free interval (TFI).

Our data showed no obvious effect of chemotherapy on MT morphological findings. The comparison of MT scoring before and after chemotherapy was not available in 4 recurrent cases. This is now under investigation to determine whether a good MT scoring change is indicative of a good biochemical response. One more problem in our scoring system is that this is only based on platinum sensitivity and it is not clear whether the score is indicative of the sensitivity to other type of widely-used drugs such as taxanes or topoisomerase inhibitors. Additionally, our group of patients did not include mucinous adenocarcinomas and we need to confirm the MT score in this relatively rare tumor type.

Finally, the number of cases in our study is small and carefully designed prospective studies in larger number of cases with clinical follow-up will be necessary to investigate more the clinical relevance of this MT scoring system. However, our data presented here provide evidence that the system could be of great value as a biomarker for chemosensitivity in OC.

\section{Acknowledgements}

We wish to thank Dr Steven G. Silverberg, Director of Anatomic Pathology, University of Maryland Medical Center, Baltimore, Maryland, USA. for critical review of this paper.

\section{References}

1. Andrews PA and Howell SB: Cellular pharmacology of cisplatin: perspectives on mechanisms of acquired resistance. Cancer Cell 2: 35-43, 1990.

2. Sorenson CM and Eastman A: Influence of cis-diamminedichloroplatinum (II) on DNA synthesis and cell cycle progression in excision repair proficient and deficient Chinese hamster ovary cells. Cancer Res 48: 6703-6707, 1988

3. Eastman A: Activation of programmed cell death by anticancer agents: cisplatin as a model system. Cancer Cell 2: 275-280, 1990.

4. Gonzalez VM, Fuertes MA, Alonso C and Perez JM: Is cisplatin-induced cell death always produced by apoptosis? Mol Pharmacol 59: 657-663, 2001

5. Isonishi S, Saitou M, Yasuda M and Tanaka T: Mitochondria in platinum resistant cells. Hum Cell 14: 203-210, 2001.

6. Hirama M, Isonishi S, Yasuda M and Ishikawa H: Characterization of mitochondria in cisplatin resistant human ovarian carcinoma cells. Oncol Rep 16: 997-1002, 2006.

7. Kim T-S, Yun BY and Kim IY: Induction of the mitochondrial permeability transition by selenium compounds mediated by oxidation of the protein thiol groups and generation of the superoxide. Biochem Pharmacol 66: 2301-2311, 2003.

8. Chilin A, Dodoni G, Frezza C, Guiotto A, Barbieri V, Di Lisa F, and Canton M: 4-hydroxymethyl-1,6,8-trimethylfuro [2,3-h] quinolin-2(1H)-one induces mitochondrial dysfunction and apoptosis upon its intracellular oxidation. J Med Chem 48: 192-199, 2005.

9. Isonishi S, Saitou M, Ochiai K, Yasuda M and Tanaka T: Enhancement of sensitivity to cisplatin by orobol is associated with increased mitochondrial cytochrome c release in human ovarian carcinoma cells. Gynecol Oncol 90: 413-420, 2003.

10. Silverberg SG: Histopathologic grading of ovarian carcinoma: A review and proposal. Int J Gynecol Pathol 19: 7-15, 2000.

11. McCluggage WG, Lyness RW, Atkinson RJ, et al: Morphological effects of chemotherapy on ovarian carcinoma. J Clin Pathol 55: 27-31, 2002.

12. Blaustein's Pathology of the Female Genital Tract. Kurman RJ (ed). 3rd edition, Springer-Verlag, Berlin, 1987. 
13. Benda JA and Zaino R: GOG Pathology Manual. Gynecologic Oncology Group, Buffalo, NY, 1994.

14. Therasse P, Arbuck SG, Eisenhauer EA, et al: New guidelines to evaluate the response to treatment in solid tumors. European Organization for Research and Treatment of Cancer, National Cancer Institute of the United States, National Cancer Institute of Canada. J Natl Cancer Inst 92: 205-216, 2000.

15. Rustin GJ: Use of CA-125 to assess to response to new agents in ovarian cancer trials. J Clin Oncol 21 (Suppl 10): S187-S193, 2003.

16. Vasey PA, Paul J, Birt A, et al: Docetaxel and cisplatin in combination as first-line chemotherapy for advanced epithelial ovarian cancer. J Clin Oncol 17: 2069-2080, 1999.
17. The International Collaborative Ovarian Neoplasm (ICON) Group: Paclitaxel plus carboplatin versus standard chemotherapy with either single-agent carboplatin or cyclophosphamide, doxorubicin, and cisplatin in women with ovarian cancer: the ICON3 randomised trial. Lancet 360: 505-515, 2002.

18. Eisenhauer EA, Vermorken JB and van Glabbeke M: Predictors of response to subsequent chemotherapy in platinum pretreated ovarian cancer: a multivariate analysis of 704 patients. Ann Oncol 8: 963-968, 1997. 